\title{
Los agricultores como actores de la política agroambiental. Un enfoque multidimensional
}

\section{Fernando E. Garrido-Fernández}

Instituto de Estudios Sociales Avanzados de Andalucía (IESA-CSIC)

fgarrido@iesaa.csic.es

\section{Resumen}

En este artículo se analizan las actitudes de los agricultores ante la política agroambiental. Para ello, se recurre a un enfoque multidimensional (económico, social y medioambiental), que permite analizar el sistema de preferencias de los agricultores ante esta política. El predominio de una u otra dimensión a la hora de acogerse a un programa agroambiental dependerá de ese sistema de preferencias y de cómo éste interrelaciona con una estructura de oportunidades en la que intervienen, además de factores sociodemográficos y estructurales, la naturaleza y el contenido de los programas y las actitudes y estrategias de la Administración y de las organizaciones profesionales agrarias (OPA).

Palabras clave: política agroambiental, actores sociales, agricultores.

Abstract. The role of farmers in the agri-environmental policy. A multidimensional approach

In this article the author analyses farmers' attitudes facing with agri-environmental policy. To this end, a multidimensional approach is chosen (economic, social and environmental) allowing to analyse the farmers' preferences framework. What dimension predominates in deciding to apply or not for an agri-environemental measure depends on that preferences framework, and on how it interacts with an opportunities structure made up of the content and nature of agri-environmental programme and the attitudes and strategies of governments and farmers' unions.

Key words: agri-environmental policy, social actors, farmers.

\section{Sumario}

1. Introducción 4. Los agricultores y el programa agroambiental

2. El sistema de preferencias de los agricultores: un enfoque pluridimensional

3. Nota metodológica

5. A modo de conclusión

Bibliografía 


\section{Introducción}

Al igual que en otros sectores de la política pública, el medio ambiente se ha introducido como un elemento a tener en cuenta en la agricultura, siendo la política agroambiental un claro exponente de esta tendencia. Los distintos actores que en ella participan —Administración agraria y ambiental, organizaciones agrarias y los propios agricultores - han ido tomando posiciones al respecto, posiciones que se han trasladado a sus discursos y estrategias - algunas veces encontradas - respecto a lo que el medio ambiente es y/o debe ser para la agricultura y cómo ponerlo en práctica a través de las correspondientes políticas.

La política agroambiental no ha sido una política creada ex-novo, sino que surgió del seno de la PAC en el contexto de su reforma en 1992, por lo que no está todavía dotada de sus propios fondos de financiación ni se formula ni se aplica a través de un marco institucional propio. Tampoco puede decirse que haya sido resultado directo de demandas sociales articuladas en nuevas comunidades de intereses, sino más bien de cambios en el orden de prioridades de la PAC una vez comprobado el agotamiento del modelo productivista promovido por ella y haberse hecho tangibles los efectos perversos del sistema de protección ligado a dicha política. La naturaleza de la política agroambiental no podría explicarse, por tanto, sin comprender el proceso de génesis, desarrollo y reforma de la PAC, ni tampoco podrían explicarse sus primeros resultados y las dificultades de su implementación sin analizar los discursos ni las estrategias de los viejos actores ligados a la política agraria (sindicatos agrarios y organismos públicos encargados de aplicarla en cada estado miembro).

Bien es cierto que los destinatarios finales de la política agroambiental son los agricultores y que es el agricultor quien, a título individual, decide libremente acogerse a un programa específico. También es igualmente cierto que en ese proceso de toma de decisiones influyen muchos factores, algunos basados en racionalidades de tipo instrumental - tales como el atractivo de la ayuda que le ofrece el correspondiente programa o el coste de oportunidad que le supone acogerse al mismo en comparación con otras opciones- y otros en racionalidades orientadas a valores —como su identificación con la existencia de programas que promuevan la introducción de métodos destinados a reducir los efectos negativos del modelo intensivo de agricultura sobre el medio ambiente.

Pero las decisiones de los agricultores respecto a la política agroambiental no son sólo el resultado de sus actitudes, valores y costes de oportunidad, sino también del entorno institucional en que tienen que desarrollar sus acciones. En efecto, una decisión favorable a un programa agroambiental puede estar motivada en última instancia por la influencia que ejercen sobre los agricultores el marco institucional del Estado — que puede ofrecer oportunidades favorables, como por ejemplo la existencia de un buen sistema de extensión agraria capaz de divulgar adecuadamente el programa y asesorar a los agricultores en la formalización y el seguimiento de los contratos- o los actores pri- 
vados de tipo colectivo — que pueden adoptar actitudes y estrategias favorables al programa y promoverlo entre sus asociados.

En definitiva, en el proceso de toma de decisiones de los agricultores respecto a la introducción en sus explotaciones de métodos más respetuosos con el medio ambiente influye no sólo lo que la teoría de la elección racional denomina sistema de preferencias — construido sobre bases de racionalidad tanto sustantiva como instrumental—, sino también lo que enfoques más cercanos al neoinstitucionalismo llaman estructura de oportunidades, para referirse al entorno en el que aquéllos desarrollan sus acciones —el contenido de la propia política agroambiental, la red de apoyo institucional, el sistema público o semipúblico de asesoramiento o la presencia de actores colectivos con capacidad para elaborar un nuevo discurso sobre las relaciones entre agricultura y medio ambiente.

Aplicando este enfoque a la realidad empírica, el que los agricultores se acojan o no a los programas agroambientales será resultado de la combinación de su sistema de preferencias y de la estructura de oportunidades en que desarrollan sus acciones. La naturaleza y el contenido de los programas, así como las actitudes y las estrategias de la Administración y de las OPA, constituyen la estructura de oportunidades de los agricultores ante la política agroambiental. En este artículo analizaremos sus actitudes y valores (sistema de preferencias) y cómo se relacionan con los elementos de dicha estructura de oportunidades, para explicar sus decisiones respecto a los programas agroambientales.

\section{El sistema de preferencias de los agricultores: un enfoque pluridimensional}

Entendiendo que el sistema de preferencias de los agricultores está formado por sus valores y actitudes ante los cambios que experimenta la agricultura y la política agraria y ante las implicaciones ambientales de su actividad, en este apartado elaboraremos un marco analítico que nos permita comprender de forma ordenada la realidad empírica en que se mueven los agricultores. Como ocurre con todo intento de construcción teórica, por muy a escala intermedia que sea, también aquí la elaboración de un marco analítico supone simplificar intencionadamente la realidad observada reduciendo su complejidad a unas cuantas dimensiones consideradas como relevantes, para, a partir de ellas, proponer algunas hipótesis explicativas.

Considerando que los programas agroambientales son parte de una política encaminada, fundamentalmente, y al menos en sus objetivos explícitos, a promover la introducción de métodos más sostenibles de agricultura, y teniendo en cuenta que la noción de sustentabilidad es una noción definida fundamentalmente a partir de tres dimensiones (económica, social y medioambiental), hay que admitir en términos teóricos que dichos programas generan en los agricultores un sistema de preferencias que puede agruparse también en tres dimensiones, lo que supone un cambio de planteamiento respecto a lo que ha sido el análisis de otras medidas de política agraria. 
En efecto, durante las décadas de 1950 a 1970, gran parte de los estudios que trataban de explicar la actitud y el comportamiento de los agricultores ante determinadas medidas de política agraria destinadas a la modernización de las explotaciones agrarias y al aumento de la productividad, optaban por basar sus explicaciones en el predominio de la dimensión económica. En estos estudios, las actitudes y los comportamientos de los agricultores ante esas medidas se explicaban por los efectos que ellas podían tener, o se esperaba que tuvieran, en el aumento de las producciones e, indirectamente, en la renta agraria.

Ya en los años ochenta, con el desarrollo de las políticas socioestructurales, se empiezan a tener en cuenta, junto con la dimensión económica, otros factores a la hora de formular las políticas agrarias, como el mantenimiento de población en el medio rural y el apoyo a las explotaciones familiares con dificultades para ser competitivas en mercados abiertos (dimensión social). Asimismo, comienzan a introducirse criterios medioambientales, dado el cariz preocupante que estaban adquiriendo en algunas zonas europeas los efectos contaminantes de una agricultura cada vez más intensiva. De este modo, y a raíz sobre todo de la reforma de la PAC en 1992, la lógica dominante en las políticas agrarias será una lógica pluridimensional en la que estarán cada vez más presentes, junto a la dimensión económica, una dimensión social (ayudar a garantizar las rentas de las explotaciones familiares) y otra ecológica o medioambiental (evitar los efectos negativos de la agricultura sobre el medio ambiente).

Si tenemos en cuenta, además, que un enfoque unidimensional que enfatice sólo la dimensión económica como motor de las decisiones de los agricultores, es insuficiente para dar cuenta de la compleja y heterogénea realidad de la agricultura en cuanto espacio de producción y reproducción sociocultural, y si consideramos la tarea plurifuncional que cada vez más se le otorga a los agricultores, creemos que una aproximación multidimensional a su sistema de preferencias es la más adecuada para el actual contexto.

Es por todo ello por lo que optamos aquí por un enfoque que no sólo considere la dimensión económica, sino también la social y medioambiental, tratando de ver qué lógica o qué combinación de ellas influye en mayor medida en la decisión de los agricultores ante los programas agroambientales. Desde esta óptica, adquiere relevancia una visión más amplia de la agricultura no sólo como un espacio de transacción económica, sino también como escenario de actividades socioculturales, interacciones ecológicas y relaciones medioambientales (Costabeber, 1998).

Dado el nivel teórico en el que nos movemos, hay que señalar finalmente que ninguna de esas tres dimensiones aparecerá de manera aislada en las actitudes y en el comportamiento de los agricultores, y que la separación que aquí se hace responde únicamente a necesidades analíticas. En la realidad empírica se producirá una combinación de varias dimensiones, pudiendo predominar alguna de ellas en unos casos o bien mantenerse las tres en posiciones equilibradas (ibídem).

A continuación, se tratará de analizar cada una de esas tres dimensiones por separado, resaltando sus elementos más significativos. 


\section{La dimensión económica}

La agricultura como actividad económica orientada al mercado está inserta en una dinámica que privilegia el uso de los recursos naturales como factores de producción de los que hay que obtener el máximo rendimiento y beneficio. Esto puede suponer un inconveniente al uso planificado de dichos recursos, de manera que puedan atender otras funciones orientadas a la preservación del medio ambiente o a la mejora de la calidad de vida (Costabeber, 1998).

Las contradicciones que se generan entre los objetivos económicos y las necesidades de renovación de los sistemas agrarios llegan a poner en peligro el mantenimiento de los niveles de producción a largo plazo y, en situaciones extremas, incluso la propia continuidad de la explotación agraria. Es decir, la racionalidad instrumental o funcional — establecida en base a supuestos netamente economicistas - se impone a la racionalidad sustantiva portadora de valores que va más allá de la mera consideración de la naturaleza como suministradora de factores de producción.

La dimensión económica, por tanto, con independencia de su influencia social o ecológica, adquiere relevancia para explicar los procesos de intensificación, incorporación tecnológica o búsqueda de nuevos nichos de mercado que pretenden alcanzar mayores niveles de competitividad en unos mercados cada vez más complejos, ya que los actores sociales inmersos en la lógica del mercado son inducidos a maximizar los beneficios económicos como forma de mantenerse en el sector. Desde este punto de vista, la dinámica impuesta a los agricultores en la búsqueda de esa competitividad en el mercado es tal, que no se tiene en cuenta si los procesos productivos son o no ahorradores de recursos naturales, deterioran o no el medio ambiente o causan desequilibrios sociales y pérdida de calidad de vida en las comunidades rurales.

La percepción de las dificultades económicas y financieras para seguir el ritmo marcado por los avances tecnológicos que hagan más competitivas las explotaciones agrarias, podría explicar la opción por unas prácticas agrarias alternativas más respetuosas con el medio ambiente. La adopción de unos estilos de agricultura que contemplen una producción ahorradora de capital y energía sería, por tanto, una estrategia motivada por esa dimensión económica.

No obstante, dentro de las estrategias de comportamiento de los agricultores marcadas por la dimensión económica, podríamos distinguir dos tipos. En primer lugar, la basada en acciones que responden a una dimensión económica, pero con una lógica mercantil, es decir, encaminadas a conseguir una mayor competitividad en el mercado o a obtener unos mayores beneficios económicos del mismo. Así, por ejemplo, aquellos agricultores que introducen técnicas más respetuosas con el medio ambiente o modelos de agricultura más sostenible por la posibilidad de negocio que les supone la aparición de un nuevo mercado que demanda los productos así obtenidos, sería una opción con una clara dimensión económica, pero con una lógica mercantil. Se podría decir, en este caso, que la decisión es más una «exigencia» del mercado, que el resultado de una actitud proambientalista (Canuto, 1998). 
Bajo esta misma dimensión económica con lógica mercantil, también tendríamos el caso de las estrategias de aquellos agricultores que, no estando sus explotaciones orientadas al mercado o en el límite de la competitividad con otras explotaciones con mejores condiciones estructurales, deciden acogerse a unos modelos de agricultura más respetuosos con el medio ambiente, con el objetivo de dar una nueva reorientación a su explotación e intentar ser competitivos en un nuevo nicho de mercado que incluiría, por ejemplo, los productos de calidad o los ecológicos. Hay que señalar, en este caso, que la ausencia de políticas agrarias públicas del tipo de la PAC europea — que garantizan el mantenimiento de los agricultores en el medio rural ejerciendo la actividad agraria a pesar de tener explotaciones poco competitivas en el mercado- podría convertir la decisión de algunos agricultores de buscar otros mercados donde ser competitivos en estrategias marcadas por una dimensión social, más que económica. Esto es, la búsqueda de estos nuevos mercados y los cambios que ello conlleva podrían estar motivados por el objetivo de poder permanecer ligados al sector agrario, manteniendo así su forma de vida. Éste es el caso descrito por Costabeber (1998) para la agricultura familiar brasileña en Río Grande do Sul, donde, en un contexto de ausencia de políticas agrarias proteccionistas, algunos agricultores han optado por un cambio hacia una agricultura ecológica como forma de seguir ligados a la actividad agraria y al medio rural.

En segundo lugar, estarían aquellas estrategias de actuación que, marcadas igualmente por una dimensión económica, estarían guiadas, sin embargo, por una lógica de compensación o complemento de rentas. Sería éste el caso, por ejemplo, de aquellas decisiones de agricultores que se inclinan por los programas agroambientales simplemente por la ayuda económica que reciben y con el único objetivo de compensar y/o aumentar la renta obtenida de su explotación. Estas ayudas serían elegidas por el agricultor de entre el conjunto de ayudas que ofrece la actual PAC y que, en un momento determinado, le resultan al agricultor más atractivas económicamente, sin plantearse en ningún momento las repercusiones ambientales que tal decisión pudiera tener.

\section{La dimensión social}

El proceso de modernización e intensificación de la agricultura ha tenido efectos positivos sobre la producción de alimentos y las rentas de los agricultores, pero también ha desencadenado efectos negativos desde otros puntos de vista, como el social o el ambiental.

El avance de la modernización y la adecuación tecnológica que han permitido explotaciones competitivas en el mercado ha dejado en el camino a un gran número de agricultores que, en su momento, no pudieron seguir el tempo modernizador. Por otro lado, esa modernización — ligada fundamentalmente a la consecución de unos elevados niveles de producción - ha supuesto un mayor consumo de productos agroquímicos (fertilizantes, pesticidas, herbicidas) por parte de los agricultores, quienes, una vez alcanzada la autosuficiencia alimentaria, están empezando a ser vistos por la sociedad europea como 
productores de excedentes, así como una muestra de despilfarro en los presupuestos nacionales y europeos (Frouws, 1998) y como causantes de externalidades más negativas - traducidas en su potencial contaminante y la «artificialización» de la producción-, que positivas. Todo ello en un momento en el que la conciencia ambiental de la población en general está adquiriendo un mayor protagonismo y en el que la información sobre los efectos negativos para la salud del uso de determinados productos en la agricultura llega con facilidad a un segmento cada vez mayor de la población.

Consecuencia de ello es que la imagen tradicional y hasta cierto punto idílica del agricultor como productor de alimentos e íntimamente ligado con la naturaleza está empezando a cuestionarse en muchos países europeos, viéndose ahora como un agente contaminante que hay que controlar y al que además se está protegiendo a través de un complejo sistema de ayudas que favorecen seguir con esos modelos intensivos.

Por otro lado, la agricultura pierde peso como sector económico en la mayor parte de los países de la UE, al mismo tiempo que disminuye su influencia como grupo de presión, sintiéndose además la necesidad de reducir el presupuesto destinado a este sector con el fin de orientarlo a la creación y al fomento de otras políticas comunes europeas. Surgen, desde la sociedad europea, nuevas demandas para el espacio rural además de la de producción de alimentos, como las de reservas naturales, zonas residenciales, parques recreativos o espacios para la captación de agua, convirtiéndose esta variada utilización del medio rural en una cuestión de interés público, que emana, entre otras cosas, de la creciente popularidad de las áreas rurales como lugar de recreo, de actividades de ocio o de retiro y del mayor interés de los consumidores por alimentos producidos de una forma sana y natural. El medio rural está, por tanto, siendo virtualmente «reformado" o "reconstruido» de acuerdo con la lógica de los sistemas agrarios, ecológicos o con las diferentes imágenes existentes sobre el paisaje (Nooij, 1993, citado en Frouws, 1998).

La imagen tradicional del agricultor, por tanto, se empieza a ver cuestionada por la sociedad europea, y necesita dotarse de una nueva legitimidad que le rehabilite frente a ella, de manera que su profesión y su labor vuelva a ser reconocida, dignificada y respetada ya no sólo como productor de alimentos, sino también como conservador del medio ambiente.

Pero, para no seguir siendo considerados responsables de la contaminación del medio ambiente y de la destrucción de la naturaleza y poder renovar su contrato con la sociedad, la comunidad agrícola debe "poner su casa en orden» (Frouws, 1998), con lo que podrá así legitimar una labor de administrador y garante de unos recursos naturales y unos valores culturales. De ahí que las prácticas agrarias con efectos perjudiciales sobre el medio ambiente o la salud (humana o animal) son percibidas como un peligro a la credibilidad de la agricultura como depositaria de esas funciones.

En este sentido, el proceso de adopción de prácticas agrarias más respetuosas con el medio ambiente, cuando es observado desde una dimensión social, puede explicarse a partir de una mayor valoración por parte del agri- 
cultor de beneficios tales como la mejora de las condiciones de trabajo mediante la reducción del uso de agroquímicos, pero también puede explicarse por la mayor legitimación social y el «autorreconocimiento» que implica la oferta al conjunto de la sociedad de productos más sanos y «limpios» elaborados a partir de unas prácticas menos contaminantes, y por la respuesta a esas nuevas demandas de la sociedad, aceptando ser los garantes del buen estado del medio ambiente y los depositarios de ciertos valores culturales que se consideran como una especie de patrimonio social.

\section{La dimensión medioambiental}

Esta dimensión se caracterizaría por una reacción frente a la degradación del medio ambiente y los recursos naturales que amenaza a la supervivencia de los agroecosistemas, tanto en su función productiva como paisajística o de ordenación del territorio.

El objetivo perseguido por las actuaciones fundamentadas en esta dimensión medioambiental sería, pues, recuperar y mantener la capacidad productiva y regenerativa del ecosistema a través de la adopción de técnicas, métodos y procesos de producción más compatibles con el respeto al medio ambiente. Su fundamento esencial sería la opción por un nuevo estilo de agricultura, con una base más ecológica para el alcance de mayores niveles de sustentabilidad agraria (Costabeber, 1998).

La opción por modelos o sistemas de producción agraria más respetuosos con el medio ambiente sería también el resultado de la percepción que tienen los agricultores de una supuesta "crisis ecológica», que se estaría manifestando en una pérdida de la capacidad de los agroecosistemas y en la creciente artificialización del sistema de producción agraria basada en el empleo de productos químicos de origen industrial. Con esta estrategia se perseguiría, por ejemplo, un cambio que diese paso a otros sistemas de producción agraria que potenciasen los recursos naturales localmente existentes.

Es decir, los agricultores, movidos por una dimensión ambiental, elegirían optar por unos métodos de producción más respetuosos con el medio ambiente por su repercusión en la mejora en el entorno ambiental y de los recursos naturales y por una concepción de la agricultura más natural y menos artificializada.

El proceso de modernización comentado en la dimensión social también tiene su incidencia en la componente ambiental, ya que el agricultor se ha visto en ocasiones arrastrado por esa espiral tecnológica para poder sobrevivir como productor y se ha quejado de que las políticas de ayudas y subvenciones han desnaturalizado en cierta manera su función, su contacto con la naturaleza y con los entresijos de la producción y su libertad como empresario para elegir qué producir. Podríamos decir que ha tenido lugar, como externalidad de ese proceso modernizador, un alejamiento o una alienación del agricultor respecto del ecosistema productivo. La política agroambiental, al promover métodos de producción más respetuosos con el medio ambiente, 
basados muchos de ellos en el respeto a los ciclos naturales, sin forzarlos o "artificializarlos», puede devolver al agricultor un sentimiento de mayor cercanía con la naturaleza, haciéndolo depositario de funciones como la recogida en la Declaración de Cork: «[...] la agricultura es y debe permanecer siendo la principal conexión entre los seres humanos y el medio ambiente, y [...] los agricultores tienen una responsabilidad como gestores de muchos de los recursos naturales del medio ambiente» (Guía Metodológica del Observatorio Europeo LEADER, 2001).

También dentro de esta dimensión medioambiental, la percepción de esa "crisis ecológica», además de esa preocupación por el deterioro del medio ambiente, puede tener igualmente unas connotaciones más ideológicas. En este caso, son cuestiones tales como el compromiso con las generaciones futuras para que hereden un medio ambiente «aceptablemente» limpio o la convicción de que las técnicas agrarias intensivas generan excedentes que inundan los mercados y hacen caer los precios, perjudicando así a los países más pobres, las que pueden motivar que el agricultor se incline por introducir en su explotación estos sistemas productivos medioambientalmente más sostenibles, ya que, de esta forma, creen contribuir a una sociedad más solidaria y justa.

\section{Nota metodológica}

La recogida de información necesaria para conocer el posicionamiento de los agricultores en la dinámica entre agricultura y medio ambiente (y, más concretamente, ante el programa agroambiental de la UE y de su aplicación en España), se realizó a través de una metodología de carácter cualitativo. En concreto, se realizaron seis grupos de discusión. Tres en la región de Castilla-León, concretamente en la zona de Villafáfila, seleccionada por ser una de las primeras zonas en las que se aplicó un programa agroambiental, concretamente el de conservación de aves esteparias (avutarda), y ofrecer, por tanto, un escenario de cierta perspectiva temporal para el análisis. El primero de los grupos se constituyó con grandes agricultores de agricultura convencional, la mayoría de ellos no acogidos al programa agroambiental; el segundo, con agricultores medianos más tradicionales, y el tercero, con agricultores medianos y grandes con orientación productiva diversa y con una buena parte de ellos receptores de ayudas por el programa agroambiental ${ }^{1}$.

1. Los grupos de discusión de Castilla y León se realizaron en las localidades de Osorno (Palencia) (grupo de grandes agricultores, de mediana edad y agricultura convencional, acogidos y no acogidos al programa agroambiental), Santa María la Real de Nieva (Segovia) (grupo de medianos agricultores, de edad avanzada y agricultura tradicional, la mayoría no acogidos al programa agroambiental), y Villarrín de Campos (Zamora) (grupo de medianosgrandes agricultores, más heterogéneo en orientación productiva y edad, acogidos y no acogidos al programa agroambiental). El trabajo de campo correspondiente a estos grupos se desarrolló a finales de 1999. 
Otros tres se realizaron en Andalucía, por ser una zona con un amplio abanico de actuaciones comprendidas en el programa agroambiental y donde se eligieron zonas de interés para la investigación. Así, un grupo tuvo lugar en una zona donde se ha aplicado con cierta intensidad el programa de agricultura ecológica en el sector olivarero y ganadero, y estuvo formado por pequeños y medianos agricultores de esos sectores (acogidos y no acogidos a las ayudas agroambientales). Otro grupo se realizó en una comarca donde está teniendo bastante éxito el programa de agricultura ecológica entre los pequeños olivareros; el tercer grupo estuvo formado por grandes agricultores de la campiña del Guadalquivir no acogidos a ningún programa agroambiental ${ }^{2}$. Mientras que con los dos primeros grupos se pretendía conocer la percepción que tienen los pequeños y medianos agricultores de los problemas a los que se enfrenta el sector agrario y la valoración que hacen de la aplicación del programa agroambiental desde sus propias experiencias como beneficiarios, con el tercero se buscaba explorar las actitudes y las opiniones de los empresarios sobre las implicaciones medioambientales de la actividad agraria.

Como complemento a estos grupos de discusión, se realizaron una veintena de entrevistas a agricultores de las zonas seleccionadas acogidos a algún programa agroambiental ${ }^{3}$. El objetivo de estas entrevistas ha sido debatir de forma más detallada algunas de las cuestiones surgidas en los grupos de discusión y conocer, directamente de los beneficiarios, el proceso de toma de decisiones que les ha llevado a acogerse a dichos programas, así como un primer balance de su aplicación.

\section{Los agricultores y el programa agroambiental}

En este apartado aplicaremos el marco analítico anterior al caso empírico que ha sido objeto de nuestro análisis, tratando de plantear algunas hipótesis explicativas sobre el comportamiento de los agricultores ante la aplicación del programa agroambiental.

2. Los grupos de discusión de Andalucía se desarrollaron en las localidades de Villanueva de Córdoba (Córdoba) (grupo de medianos ganaderos y agricultores, menores de 50 años, unos convencionales y otros acogidos al programa agroambiental de agricultura/ganadería ecológica), Puente Génave (Jaén) (grupo de pequeños agricultores de olivar, mayores de 50 años, receptores y no receptores de ayudas del programa agroambiental) y Córdoba (grupo de grandes agricultores convencionales de campiña, menores de 50 años y no acogidos al programa agroambiental). Los grupos se realizaron en el primer semestre del año 2000.

3. Las entrevistas complementaron la información recogida a través de los grupos y fueron realizadas entre los años 2000 y 2001. La información ha sido actualizada posteriormente por el autor a través de su colaboración en el proyecto de investigación «Elaboración de una metodología para el control y seguimiento de los programas agroambientales y de forestación en explotaciones agrarias en la Comunidad Autónoma de Andalucía(1999-2003)», desarrollado por la ETS. de Ingenieros Agrónomos y de Montes de la Universidad de Córdoba bajo la dirección de la Dra. Pilar Fernández Rebollo y financiado por la Consejería de Agricultura y Pesca de la Junta de Andalucía. 


\section{El predominio de la dimensión económica en las estrategias de los agricultores}

A partir del análisis de los grupos de discusión y de las entrevistas realizadas a los agricultores, hay un cuestión que se hace evidente: la necesidad de conservar el medio ambiente tiene para el agricultor un límite claro, el hecho de que la naturaleza es también la base de su actividad productiva. Esta oposición entre, por un lado, la necesidad de conservar y, por otro lado, la de explotar la tierra en tanto que su principal factor de producción, se sitúa en el centro de la relación del agricultor con el medio ambiente, relación que suele inclinarse a favor de la actividad productiva:

[...] es que es un arma de doble filo: si queremos proteger la fauna y vamos a abandonar la agricultura [...] Nosotros dependemos de la agricultura por encima de lo otro. (Grupo de discusión de Génave)

Estas dos facetas contrapuestas que muestra el agricultor se acentúan en cierto modo por la creciente consideración de su actividad como empresarial, tanto entre los grandes como entre los pequeños agricultores. En los últimos años, y particularmente desde la entrada de España en la UE, el agricultor parece haber cambiado la concepción de la actividad que realiza. Para el agricultor, su actividad ya no persigue exclusivamente su subsistencia, sino que tiene ahora un cariz eminentemente empresarial del que no está ausente ninguna de las consideraciones económicas que rigen para cualquier otra empresa en otro sector.

Como decimos, la entrada en la UE ha agudizado la necesidad de competir más y mejor. Ser suficientemente competitivo es la única manera que tiene el agricultor de asegurarse una posición en el actual mercado agrícola; de lo contrario, podría perder beneficios o cuota de mercado o bien verse expulsado de la actividad agraria en el extremo más desfavorable. Esta preponderancia de la competitividad se traduce en un protagonismo de la dimensión económica en la mayor parte de las estrategias que el agricultor adopta respecto de su explotación y que, por lo tanto, está presente en una buena proporción en la decisión del agricultor de acogerse a las medidas agroambientales que aquí se analizan.

Para algunos agricultores, no obstante, la toma de conciencia de los problemas ambientales relacionados con la agricultura se produce al constatarlos en su explotación y en la influencia negativa que los mismos tienen sobre los rendimientos (erosión, agroquímicos, uso del agua...). La utilización de métodos o técnicas agrarias más respetuosas con el medio ambiente se justifica en estos casos como una manera de recuperarlo como un conjunto de factores productivos y, como consecuencia de la adopción de estas técnicas — que en muchos casos implican menor uso de inputs-, también por el abaratamiento de los costes de producción, que se traduce al mismo tiempo en una mayor competitividad.

Este comportamiento, además del propio beneficio sobre la explotación, puede venir acompañado de otro factor favorable y que también es tenido en 
cuenta por algunos agricultores. Éste es el hecho de que los productos así obtenidos pueden calificarse comercialmente de «más naturales» $\mathrm{O}$ "producidos respetando el medio ambiente», característica ésta que les permite obtener una mayor aceptación por un segmento del mercado y unos precios más altos que estimulan su producción. Este fenómeno se muestra más claro para el caso de la ayuda a la agricultura ecológica, donde una buena parte de los que optan por este sistema de producción lo hacen con vistas a conquistar un mercado emergente, pero cada vez más importante y que puede proporcionar beneficios económicos interesantes:

Desde la cooperativa se ha intentado hacer llegar a los socios la idea de que (la ayuda agroambiental a la agricultura ecológica) es una solución rentable para este olivar de montaña, ya que se obtienen unos precios casi un 50\% mayores que los del producto tradicional. Hemos intentado destacar su rentabilidad independientemente de la ayuda que existe para ello. (Entrevista realizada a un técnico de cooperativa ecológica y agricultor de Pozoblanco, Córdoba)

Pero, de las entrevistas realizadas, se pueden distinguir dentro de este comportamiento dos tipos de agricultores que, si bien guían sus estrategias por una lógica económica, se corresponden con distintas condiciones de partida. Para un tipo de agricultor (moderno, competitivo y con altos niveles de formación), los programas agroambientales representan una oportunidad de reducir costes en sus explotaciones y aumentar sus niveles de competitividad. Para el segundo tipo de agricultor (con explotaciones no modernizadas y con hándicaps estructurales), los programas agroambientales son una oportunidad para no ser excluidos económicamente y poder complementar sus rentas.

\section{a) El programa agroambiental como oportunidad para introducir una nueva racionalidad en explotaciones modernas y competitivas}

En el primer nivel, tendríamos a un agricultor con una explotación moderna, viable económicamente y orientada al mercado, que decide introducir prácticas agrarias más respetuosas con el medio ambiente con el objeto de mejorar los factores productivos de los que depende su capacidad productiva (suelo, materia orgánica, agua...), al mismo tiempo que reducir la utilización de inputs y, con ello, los costes de producción. Mediante esta actuación, consigue obtener igualmente un "plus» de competitividad en el mercado.

En algunos casos, esta toma de conciencia por parte del agricultor sobre los efectos perjudiciales de ciertas prácticas agrícolas intensivas sobre los recursos naturales ${ }^{4}$-recursos naturales como factores de producción, no lo olvi-

4. Podría argumentarse que la decisión del agricultor de introducir en su explotación prácticas agrarias más respetuosas con el medio ambiente cuando ha tomado conciencia de los efectos negativos de los métodos intensivos sobre los recursos naturales, responde más a una dimensión medioambiental — de recuperación del medio ambiente-, que económica. Sin embargo, la consideración que el agricultor hace del medio ambiente como fuente de sus factores productivos y su deseo de recuperarlo o conservarlo para no ver comprometidas 
demos-, les ha llevado a buscar nuevas técnicas que, sin perjudicar ostensiblemente los rendimientos de la explotación ni su capacidad productiva (dimensión económica), les permitan producir salvaguardando en lo posible los recursos naturales; es decir, procurar un uso más racional de los recursos naturales.

Un ejemplo de ello lo constituyen algunos de los agricultores entrevistados que pertenecían a la asociación Agrofuturo ${ }^{5}$. Era ésta una asociación sin ánimo de lucro de la que han formado parte miembros destacados de la comunidad científica española dedicada a las ciencias agrarias, empresas e industrias vinculadas con el sector, organizaciones agrarias, instituciones y socios individuales y que perseguían la gestión integrada de cultivos como un sistema de producción agraria que armoniza las exigencias técnicas y económicas del agricultor con el respeto al medio ambiente y la conservación de la naturaleza y el paisaje. Aunque la agricultura integrada no fue objeto de ayuda por parte del primer programa agroambiental de la UE, no cabe duda de que constituye una iniciativa de producción agrícola más respetuosa con el medio ambiente y que se adapta perfectamente a las necesidades del grupo de agricultores antes descrito, que buscan recuperar y después mantener el medio ambiente como fuente de sus factores productivos. Dado, además, que este modelo de agricultura integrada defiende, entre otras cosas, el uso de técnicas modernas y de producción rentables, es visto como una alternativa más atractiva para estos agricultores que otro tipo de prácticas (algunas de ellas incluidas en el programa agroambiental) que, persiguiendo los mismos fines de preservar los recursos naturales, podrían condicionar más los resultados de su explotación. En este orden de cosas, uno de los socios de Agrofuturo entrevistados afirmaba:

[...] he visto fotografías aéreas de mis tierras de diferentes años y he ido observando cómo la tierra ha ido cambiando de color como consecuencia de ese proceso de mineralización, con la consiguiente deficiencia en materia orgánica. Empecé a introducir algunas prácticas menos intensivas en cuanto a labores y al uso de inputs. Estas labores suponen un ahorro, aunque mínimo [...] Además, [...] no suponen un recorte en los rendimientos de la explotación. (Entrevista realizada a un agricultor de Córdoba)

El sistema de preferencias de estos agricultores se encuentra ante una estructura de oportunidades marcada no por los elementos antes señalados (contenido del programa agroambiental, actitudes institucionales de la Administración agraria y de las OPA), sino por elementos de su entorno que ellos controlan más directamente debido a su capacidad económica y a su nivel de formación. En efecto, siguiendo con el ejemplo anterior, la opción de los agricultores de la extinta Agrofuturo por métodos más sostenibles de agricultura no es resultado

en el futuro su capacidad productiva y la rentabilidad y competitividad de su explotación, nos inclinan a incluir este comportamiento bajo la dimensión económica y no ambiental.

5. Esta asociación ha desaparecido en la actualidad, si bien su modelo de funcionamiento y fines básicos pueden encontrarse en otras asociaciones similares, como la Asociación Española de Agricultura de Conservación/Suelos Vivos (AEAC/SV), que tiene su sede en Córdoba. 
de la influencia de la estructura de oportunidades antes comentada, sino de su interacción con iniciativas surgidas de los centros de investigación científica (departamentos universitarios de agronomía, institutos del CSIC, centros de investigación agraria) con los que suelen mantener estrechas relaciones de colaboración. Aunque la mayoría de estos agricultores están relacionados con las OPA, especialmente con ASAJA, con la que comparten el discurso de «capitalismo verde» ${ }^{6}$, la realidad es que poca incidencia tienen las actitudes y las estrategias del movimiento sindical agrario sobre el comportamiento de aquéllos. Puede decirse que sus decisiones y comportamientos responden a una lógica relativamente autónoma respecto de la estructura institucional de oportunidades a que hemos hecho referencia.

Dentro de este grupo de agricultores con explotaciones modernas, viables económicamente y orientadas al mercado, podríamos ubicar también a aquellos que optan por prácticas agroambientales no con la vista puesta en frenar el deterioro del medio ambiente como recurso productivo, sino con el de obtener mayor rentabilidad con unos productos más "ecológicos», ocupando así un mercado de productos sanos, de calidad y naturales, que se muestra interesante desde el punto de vista comercial. Las expectativas presentes y futuras de un mercado latente y prometedor para productos ecológicos o «sanos», han ejercido influencia en las decisiones de estos agricultores, en el sentido de iniciar una producción cualitativamente diferenciada capaz de aumentar sus niveles de competitividad y de ampliar los ingresos de la explotación agraria. Este grupo de agricultores también presenta un cierto grado de autonomía en su comportamiento, puesto que no depende de las oportunidades que les ofrece ni la Administración, ni las OPA, ni el propio programa agroambiental. Es más, para este grupo, una presencia institucional deficiente puede ser un inconveniente para emprender acciones hacia nuevas orientaciones y formas de gestión, y tener incluso efectos disuasorios:

Empecé en ello [ganadería ecológica] pensando en que sería un sector con futuro y donde podía haber un mercado interesante. [...] Pensé en el beneficio económico que obtendría al poder vender los terneros más caros, pero, al no haber aún un mercado para estos productos, los estoy vendiendo al mismo precio que los tradicionales [...] no merece la pena tantos inconvenientes para no tener un sobreprecio en el producto final, así que volveré a la explotación tradicional cuando pasen los cinco años de contrato. (Entrevista realizada a un ganadero de Villanueva de Córdoba)

\section{b) Los programas agroambientales como oportunidad para evitar la exclusión económica y para complementar rentas}

En este tipo se incluirían ciertos agricultores que, con unas explotaciones en el límite de la viabilidad económica o con unos handicaps estructurales impor-

6. El discurso del «capitalismo verde» se caracteriza por defender la protección del medio ambiente para asegurar que continúe proporcionando en el futuro recursos productivos. 
tantes, pero potencialmente competitivas, deciden optar por las prácticas agroambientales como una forma de salir de esa situación e incorporarse al «tren» de la competitividad. Para este grupo de agricultores, la posibilidad de obtener productos diferenciados — más sanos, naturales o ecológicos- no le es atractiva sólo por el hecho de obtener mayores beneficios económicos, sino sobre todo porque les da la oportunidad de ser competitivos en un nuevo mercado aún no saturado y en el que ellos se consideran capaces de poder competir. Su motivación, por tanto, está marcada igualmente por la dimensión económica, pero existe un matiz respecto al grupo anterior en cuanto a las razones que les mueven a optar por este tipo de prácticas agroambientales.

Este segundo tipo se localiza claramente en algunos agricultores situados, por ejemplo, en zonas de montaña, donde los costes de producción y los rendimientos de sus cultivos les impiden competir adecuadamente con otras explotaciones con la misma orientación de cultivos, pero situadas en zonas de campiña. Por consiguiente, las dificultades de competir en un sector de producción que, dadas ciertas condicionantes y limitaciones estructurales, no les permite continuar, puede significar en estos casos la búsqueda de nuevas orientaciones productivas en sus explotaciones, orientaciones productivas que les ofrezcan diferenciar sus productos de la competencia y buscar un nuevo valor añadido:

La agricultura ecológica [en olivar] es una solución muy buena para esta zona, que debe centrarse en la calidad y no en la cantidad. Esta zona debe apostar por la diferencia en la calidad del producto. Poco margen de maniobra y unos costes muy ajustados nos impiden ser competitivos en el olivar tradicional. Por ello, el olivar ecológico puede ser una posibilidad de futuro en esta zona. (Entrevista realizada a un agricultor de Villanueva de Córdoba)

Costabeber, en su trabajo sobre Rio Grande do Sul (1998), mostraba la existencia de este grupo de agricultores, señalando cómo los elevados costes y riesgos económicos de seguir la dinámica de tecnificación agraria convencional aparecen como uno de los determinantes fundamentales en la búsqueda de estilos de producción agraria ahorradores de capital y energía. Pero también las expectativas de inserción en un mercado distinto que remunera mejor la producción ecológica, así como las perspectivas optimistas respecto a una futura expansión del mercado para productos sanos, constituyen una importante razón que expresan los agricultores a la hora de justificar su adopción de técnicas más respetuosas con el medio ambiente en sus explotaciones.

En este grupo de agricultores sí parece que tenga influencia la estructura institucional de oportunidades antes señalada a la hora de decidirse a acogerse a algún programa agroambiental. En primer lugar, la propia divulgación del programa constituye un elemento importante, ya que, a diferencia de los empresarios agrícolas antes citados, para estos otros, de menor nivel de cualificación y de menor facilidad de acceso con sus propios recursos a las fuentes de información, la presencia de actores intermedios es fundamental, si bien ello dependerá del nivel y la calidad de servicios que le presten. Del análisis realizado se deduce que estos agricultores reconocen la importancia de orga- 
nismos como el Servicio de Extensión Agraria o las propias OPA, pero también reconocen que la actuación de estos organismos son fruto más de iniciativas aisladas e individuales de ciertos agentes, que de una actuación programada desde la Administración o los sindicatos para responder a los nuevos retos planteados por los programas agroambientales. En este sentido, se muestran críticos con la escasa calidad de la información que reciben, y señalan como más efectivos los servicios que, en ocasión de necesitarlos, les presta el movimiento cooperativo.

Pero la dimensión económica en la decisión del agricultor de acogerse a alguna de las ayudas agroambientales se refleja también en los casos en que el agricultor no busca específicamente los beneficios económicos de un nuevo mercado o una reducción de costes que le haga más competitivo, sino que la adopción de prácticas agrícolas más respetuosas con el medio ambiente responde principalmente a que resulte suficientemente atractivo el montante de la ayuda que establece el programa. En este caso, nos podemos encontrar, por un lado, a agricultores que no ejercen la actividad agraria como principal y que se orientan hacia una producción $\mathrm{u}$ otra en función de las ayudas que ofrece la PAC de la $\mathrm{UE}^{7}$ y de las menores complicaciones que les supone para la gestión de su explotación:

Desde un primer momento vi claro que aquello era interesante desde el punto de vista económico y que permitía una gestión más cómoda de la explotación. (Entrevista realizada a un agricultor de Villalón de Campos, Valladolid)

Por otro lado, también podemos encontrar agricultores que, aunque tengan la actividad agraria como principal, son muy dependientes de las ayudas de la PAC a las que han venido acogiéndose desde hace años, bien por sus condiciones socioeconómicas (endeudados, jubilados...) o bien porque las características de su explotación y las condiciones orográficas o climatológicas de la zona en que vive les resultan poco favorables para plantearse la posibilidad de cambios en las prácticas agrarias o en la orientación productiva de su explotación y, por consiguiente, la posibilidad de embarcarse en la búsqueda de nuevas iniciativas de productos y mercados. En este caso, las ayudas agroambientales son consideradas como una subvención más de la PAC que en estos momentos puede resultar interesante desde el punto de vista económico y que no les complica excesivamente los cultivos y las labores que tradicionalmente venían haciendo. Incluso en medidas que pudiéramos llamar «más comprometidas» — por los cambios en las técnicas agrícolas y los requisitos más estrictos a cumplir-, como la de conversión a la agricultura y ganadería ecológi$\mathrm{ca}$, es posible encontrar este posicionamiento, sin ninguna referencia a los posibles beneficios ambientales que estas prácticas podrían ocasionar.

7. Este tipo de comportamiento de sembrar aquello por lo que se obtiene una mayor ayuda económica de la Política Agrícola Común era calificado muy gráficamente por uno de los agricultores asistente a uno de los grupos de discusión como "plantar subvenciones». 
Como hemos indicado anteriormente, en estas circunstancias prevalece también la dimensión económica en el comportamiento de los agricultores, si bien con una lógica orientada a completar o compensar las rentas obtenidas de la explotación agraria. Las razones económicas que manifiestan los agricultores aparecen vinculadas entonces a la obtención de un complemento de renta con una escasa carga de gestión de la explotación (sobre todo para el caso de aquéllos que no tienen la actividad agraria como principal) o a lograr un nivel de renta y de ingresos que les permita continuar en la actividad agraria y no ser excluido de la misma (que sería más frecuente en las circunstancias de déficits estructurales o de competitividad y de no poder embarcarse en nuevas orientaciones para sus tierras). En este segundo caso, la dimensión económica parece mezclarse con la dimensión social, al estar vinculada a esa decisión un deseo de permanecer vinculado a la actividad agraria y al tejido socioeconómico de la zona.

A raíz de estos resultados, parece claro que los agricultores que optan por los programas agroambientales con una lógica económica de compensación y complementariedad de rentas, necesitan de la estructura institucional de oportunidades, debido, en su mayor parte y salvo excepciones, a la precariedad de sus situaciones y al bajo nivel de acceso que tienen a las fuentes de información con sus propios recursos. De los elementos de dicha estructura, es evidente que el contenido del programa ejerce una influencia fundamental a través del mayor o menor atractivo que tienen las ayudas en comparación con otras posibles, así como a través de los cambios que implica el programa en la gestión de sus explotaciones. En este grupo, la importancia del servicio de asesoramiento que puede prestarle la Administración o las OPA es fundamental, no sólo al comienzo del periodo de contrato, sino a lo largo del mismo, ya que son muchas las incertidumbres que le puede generar y la necesidad de un apoyo continuado.

\section{La dimensión social como fuente de legitimidad en el discurso de los agricultores}

Al definir las características de la dimensión social, señalábamos cómo, en los últimos años, la sociedad ha venido cuestionando la figura del agricultor por los efectos negativos que el modelo productivista ha ocasionado sobre el medio ambiente.

El agricultor es consciente de esta circunstancia, pero su actividad productiva opera directamente sobre el medio ambiente, hecho que inevitablemente produce efectos que pueden ser perjudiciales. Algunos de los agricultores intervinientes en los grupos de discusión llegan a describir su relación con el medio ambiente como una relación de amor y odio. Amor en cuanto a que el agricultor vive en y del medio ambiente y lo quiere conservar, mientras que el odio surge por una actuación político-administrativa e ideológica que entienden irracional y agresiva en la medida en que puede amenazar su subsistencia y libertad como agricultores, pues no da opción a una alternativa que permita la convergencia de la acción del agricultor y la protección del 
medio natural. Por tanto, y por regla general, el agricultor considera el medio ambiente como su entorno y medio de vida y se siente agredido por el discurso conservacionista que no tiene en cuenta este hecho y que lo señala como culpable del deterioro ambiental:

Yo creo que hay que defender el medio ambiente, hay que protegerlo, tiene que estar todo bonito [...]. Ahora, lo que no puede este tema es echarse encima de la agricultura y acabar con ella. Entonces, como el medio ambiente no se entiende desde ese punto de vista, sino que todo son limitaciones, todo son sanciones y todo son entorpecimientos. Y como, además eso va a limitar cada vez más y más, y a condicionar más la agricultura con el medio ambiente, pues entonces la relación se convierte en odio. $\mathrm{O}$ sea, que es un tema que está estorbando la propia subsistencia de los agricultores, y eso es lo verdaderamente grave. (Grupo de discusión de Córdoba)

Por otro lado, la percepción que el agricultor ha tenido hasta ahora de medidas ambientales que afectan a la agricultura, ha venido más de la Administración ambiental y en general en forma de limitaciones y/o prohibiciones -Administración a la que, además, acusan de no haber tenido en cuenta su opinión y haber considerado sólo el discurso ecologista, un discurso que ellos consideran fundamentalmente urbano, que los acusa de dañar y perjudicar al medio ambiente-, que de la Administración agraria, por lo que su primera reacción suele ser de recelo y defensiva. No se puede, según los agricultores, abogar por un sistema de conservación a ultranza del medio ambiente sin tener en cuenta que la tierra es para el agricultor ante todo un bien económico susceptible de explotación. Sólo desde esta perspectiva se podría trabajar en una síntesis entre producción y un menor daño al medio ambiente.

Frente a esta acusación de la sociedad, el agricultor también argumenta que él es víctima de las políticas. Políticas que, en los años sesenta y setenta, le impulsaron a intensificar su explotación buscando la máxima producción y sin reparar en el medio ambiente, y políticas que, a finales de los ochenta y en los noventa, promueven reducir las producciones y buscar alternativas de prácticas agrarias más respetuosas con el medio ambiente. Un victimismo que ahora extienden al mercado, que les obliga a mantener la competitividad y la productividad si quieren sobrevivir como agricultores. Y producir con esas condiciones supone, para muchos de ellos, dañar el medio ambiente.

Para producir lo que realmente el ciudadano quiere, estás obligado a utilizar ciertos productos que están en el límite de lo que es agresivo para el medio ambiente. (Grupo de discusión de Córdoba)

Ese mercado y las demandas de la sociedad suponen para el agricultor una cierta contradicción. Aducen los agricultores que se les exige que produzcan de acuerdo con los imperativos del mercado, pero sin dañar el medio ambiente y ofreciendo productos naturales. Se ven obligados, por un lado, a utilizar agroquímicos para obtener un producto con unas determinadas característi- 
cas - que son las que demanda el consumidor y el mercado- pero, por otro lado, ese mismo consumidor exige que se respete el medio ambiente. La sociedad quiere lo natural (esa es la teoría), pero genera un mercado que sólo recompensa lo sintético (esa es la práctica):

[...] pero el supermercado no lo quiere porque el consumidor no lo quiere, pero sí que dice que no puedes hacer una serie de cosas. Entonces, cómo puedo yo coger un producto hortícola con unas características sin echar una serie de productos, que están homologados... Y, además, hay una serie de empresas que para comprarte te exigen que les digas los productos y en qué dosis los has tratado para comprártelo, ¿̇ómo lo haces?. Pues no sé, pero te están exigiendo que no hagas una serie de cosas y no quieren las otras. Yo ahí me pierdo un poco. (Grupo de discusión de Córdoba)

Pero, al margen de ese malestar del agricultor frente a las imposiciones de la sociedad, parece mostrar también su desencanto por su incapacidad para trasladar a esa misma sociedad los efectos positivos que la agricultura tiene sobre el medio ambiente y el papel que la actividad agraria puede desempeñar ante esas nuevas demandas de productos y servicios. El agricultor se sitúa alejado de esa posición adoptada por la sociedad respecto a los temas ambientales que lo califica de «destructor del medio ambiente» y que, sin embargo, no le reconoce las funciones positivas que hasta ahora ha venido realizando, incluidas por supuesto las de conservación y cuidado del medio ambiente. En este sentido, creen necesario rehabilitar la imagen del agricultor en relación con esas tareas de conservación:

[...] no sabemos defender nuestra imagen y eso yo lo veo un fallo, cuando además yo creo que se da la circunstancia de que los grandes parques naturales $[\ldots]$ nos los deben a los agricultores. (Grupo de discusión de Córdoba)

[...] porque los ecologistas somos nosotros que lo tenemos aquí porque lo hemos conservado a lo largo de nuestra vida. (Grupo de discusión de Villarrín)

Es decir, que lo que está ahí bien conservado y los paisajes que ahora se quieren conservar, son fruto de la actividad agraria, y están ahí porque los agricultores lo han hecho posible. El problema es que no han sabido transmitir ese activo, y por el contrario sí se extiende el factor negativo de la contaminación que provocan. Deben, pues, defender y legitimar su trabajo.

Las medidas agroambientales, en alguna medida, vienen a posibilitar un acercamiento entre las nuevas demandas que la sociedad hace de los espacios rurales en general y de la agricultura en particular, al mismo tiempo que, acogiéndose a ellas, el agricultor puede «limpiar» su imagen de agente contaminante y legitimar su profesión. En este sentido, y en la medida en que la introducción de unos modelos de agricultura más respetuosos con el medio ambiente permiten ofrecer a la sociedad unos productos menos artificiales y más sanos, se pone de manifiesto en la decisión del agricultor una compo- 
nente o dimensión social ligada a la valoración de su trabajo a través del que obtiene productos de calidad; supondría, pues, un reconocimiento del agricultor como un productor que cuida la calidad frente a lo que vienen percibiendo como un menoscabo y un deterioro de su figura en una sociedad eminentemente urbana.

Hay que señalar también, no obstante, que el cambio que se produce hacia métodos de producción más respetuosos con el medio ambiente, si bien persigue una forma de reconocimiento social a la labor del agricultor que suministra alimentos sanos, también supone un cierto riesgo económico que, en algunos casos - aquéllos en que los cambios en el sistema de producción y la tecnología son importantes-, el agricultor no siempre está dispuesto a asumir si no se garantiza su supervivencia económica. Aquí surge la demanda de una correcta remuneración de sus productos de mayor calidad vía precio o bien una compensación o ayuda del Estado por ese cambio que beneficia a la sociedad en su conjunto.

En estos casos, la imbricación entre la dimensión social y económica no se oculta, ya que aunque las expectativas que tienen los agricultores respecto a la remuneración que recibirán por la oferta de un producto más sano o ecológico no se evalúe directamente en términos monetarios, sí se supone que tendrán alguna forma de compensación y de reconocimiento social que, en alguna medida, podrá volverse económica (Costabeber, 1998). O, dicho de otra forma, la comercialización y venta de unos productos más sanos representaría que el consumidor reconoce el bien público que está adquiriendo, con lo que valorizaría no sólo al producto sano, sino también al propio productor, es decir, al agricultor, dignificando así la labor de esta profesión.

Del trabajo empírico realizado, parece que la dimensión social sólo está presente en términos discursivos entre los agricultores, pero no parece que esté guiando su comportamiento y sus decisiones respecto a los programas agroambientales. Es un hecho evidente que, en una agricultura como la europea, en la que los agricultores se encuentran todavía bien protegidos por la política agraria, su reproducción social no parece que esté seriamente amenazada, a pesar de los cambios que se han producido en la PAC y los que se prevén en un futuro inmediato. En ese contexto, se comprende que los agricultores españoles no vean todavía en la nueva política agroambiental una vía para garantizar su reproducción social, y que la vean con un cierto recelo. Sólo en términos discursivos, en efecto, puede apreciarse una valoración positiva de la política agroambiental en tanto que una oportunidad para conectar mejor con las nuevas demandas de la sociedad.

En este sentido, parece que existe conexión entre el discurso de algunas organizaciones, como UPA y COAG, y el de ciertos grupos de agricultores familiares, que, por su mayor vinculación territorial con los espacios rurales, ponen énfasis en esta dimensión social de la política agroambiental. No se observa dicha conexión con organizaciones como ASAJA, ni tampoco con el discurso oficial de la Administración, que apenas insisten en esta dimensión. 


\section{La dimensión medioambiental, tan cerca y tan lejos de los agricultores}

Pese a ser el medio ambiente una parte del adjetivo que acompaña a las medidas aquí analizadas — medidas agroambientales—-, esta dimensión ambiental está poco presente tanto en el discurso como en la lógica que inspira sus decisiones a la hora de acogerse o no a algunas actuaciones que recoge el programa agroambiental.

En efecto, el tema del deterioro de los recursos y las implicaciones medioambientales de la agricultura tal como se plantean en los programas agroambientales, se manifiesta de un modo lejano y vago en el discurso de los agricultores entrevistados. La dimensión ambiental sólo aparece como algo cercano a los agricultores cuando se concreta en problemas ecológicos que afectan a su sistema productivo. Los problemas de erosión de suelos y los efectos de los productos químicos sobre los recursos naturales, así como el declive en la productividad de los agroecosistemas, son citados por los agricultores en muchos casos en que ha habido cambios en las prácticas productivas. El agricultor, por tanto, conecta con el discurso de la conservación del medio ambiente en la medida en que percibe directamente los daños en su entorno y, más concretamente, en su propia explotación:

Con el paso del tiempo, me he ido concienciando de que se estaba sobrexplotando el suelo y que había que conservar este recurso y cuidarlo si no quería esquilmarlo y poder dejarle a mis hijos una tierra en buenas condiciones. Las pendientes en que está situado muchas veces el olivar conduce a procesos erosivos y pérdida de suelo si no se tienen los cuidados adecuados. (Entrevista realizada a un agricultor de Córdoba)

Aunque como vimos al tratar la dimensión económica, estas actuaciones para detener el deterioro de los recursos responden más al deseo de evitar su pérdida como factores productivos, no podemos obviar que existe una cierta influencia también de la dimensión ambiental en este comportamiento, al menos en lo que se refiere a la toma de conciencia de los efectos negativos sobre el medio ambiente de ciertas prácticas agrarias y la decisión de hacer algo al respecto.

La toma de conciencia de los daños producidos a la naturaleza por algunas de las prácticas agrarias lleva al agricultor en ocasiones a dar una nueva orientación a su explotación. En este caso, la dimensión ambiental prevalece claramente en la decisión de estos agricultores de acogerse a algunas de las opciones del programa agroambiental, inclinándose la mayor parte de ellos por la agricultura ecológica. En base a las entrevistas realizadas y a los grupos de discusión, estos agricultores no suelen tener la agricultura como actividad principal y, en general, poseen una formación cultural mayor y un buen acceso a la información.

A estos factores hay que sumar también, en ocasiones, cierto compromiso ideológico; es decir, la adopción de estos métodos más respetuosos con el medio ambiente supone para estos agricultores su contribución personal — su «granito de arena»— a la causa de evitar la destrucción del planeta: 
[...] verdaderamente, puedo decir que no estoy conforme con la forma que tenemos los agricultores de actuar, que no respetamos la naturaleza, atropellamos muchas cosas. Yo estoy metido en un proceso de agricultura ecológica y estoy respetando muchas cosas del medio ambiente, y por eso quisiera que todos los agricultores se dieran cuenta de eso. ¿̨or qué?, pues porque nos estamos quedando sin pájaros en el campo, los animales naturales del campo están desapareciendo, esto es por culpa de que estamos atropellando la naturaleza. (Grupo de discusión de Génave, Jaén)

En otros casos, la adopción de prácticas agrarias más respetuosas con el medio ambiente parece responder no sólo a una mayor preocupación por la naturaleza, sino también por un menor daño para la salud. La salud es, pues, un significante que empieza a incorporarse en el discurso de algunos agricultores, de manera que la preocupación por el medio ambiente no tiene entonces tan sólo el propósito de conservarlo, sino también de garantizar la salud humana.

Finalmente, hay otra componente de la dimensión ambiental que se deja entrever en algunas de las entrevistas realizadas y que tiene que ver con el proceso modernizador a que aludíamos al tratar la dimensión social. En la medida que dicho proceso ha artificializado la producción y ha incrementado considerablemente el volumen de inputs (energía, productos químicos, etc.), ha contribuido igualmente a que algunos agricultores sientan una especie de «alienación» respecto del proceso productivo. Un proceso que ahora parece depender menos de su conocimiento de la naturaleza y de sus labores al haberse artificializado en gran medida. En estos casos, la estrategia del agricultor de introducir en su explotación prácticas agrarias más respetuosas con el medio ambiente está imbuida de una dimensión ambiental, ya que supone para él un reacercamiento a las prácticas culturales tradicionales que considera más naturales e, indirectamente, una relación más directa con el medio ambiente:

[...] el agricultor está adquiriendo una conciencia ambiental que antes no mostraba. Es como si le devolviesen a la gente la esencia de ser agricultor. (Entrevista realizada a un técnico de cooperativa en Pozoblanco, Córdoba)

La dimensión ambiental aparece en el discurso y en las estrategias de los agricultores de un modo ambiguo y contradictorio: cuando los temas agroambientales se definen en términos generales, aparecen en un lugar bastante alejado de su núcleo discursivo, pero cuando se definen haciendo referencia a problemas concretos, la dimensión ambiental emerge en un lugar central no sólo del discurso, sino también de las estrategias de los agricultores, especialmente en el caso de agricultores cuyas explotaciones están ubicadas en zonas donde son ya tangibles problemas de deterioro medioambiental.

En este sentido, puede cobrar un interés fundamental el papel de la Administración y las OPA como actores intermedios, ya que es precisamente su labor de divulgación del programa y de concreción del contenido de las distintas medidas para hacer que respondan a problemas agrombientales cerca- 
nos a la realidad de los agricultores, la que puede incentivarlos para que se acojan a dichos programas. En este punto, nos encontramos con el problema de la desigualdad de oportunidades en la que se encuentran los agricultores según la zona donde estén ubicadas sus explotaciones, debido a la desigual presencia institucional de la red de servicios de la Administración y de las OPA y a la también desigual eficacia en el desarrollo de tales servicios.

En los casos en que la decisión de acogerse al programa agroambiental está respaldada por un compromiso "ideológico», este protagonismo de la Administración y/o las OPA pasa a un segundo plano, ya que la iniciativa del agricultor no es producto de la información recibida o de la existencia de la medida agroambiental, sino de su propia concienciación sobre los problemas existentes. En este sentido, la Administración o las OPA sí pueden desempeñar el papel de prestador de los servicios que dichas iniciativas demanden.

\section{A modo de conclusión}

El análisis realizado en este artículo nos permite avanzar algunas conclusiones.

En primer lugar, se ha observado que, en el sistema de preferencias de los agricultores respecto a la política agroambiental, el principio dominante es el de valorar los programas en tanto que oportunidades para obtener rentas. Es la dimensión económica la que domina de un modo general en las preferencias de los agricultores, y tienen una presencia muy escasa, por no decir nula, otras dimensiones, como la social o la medioambiental.

En segundo lugar, que, contrariamente a lo que pudiera esperarse, parece que, cuando está presente, la dimensión ambiental muestra mayor presencia en el sistema de preferencias de los grandes agricultores que en el de los pequeños agricultores familiares. Dada la elevada magnitud de la renta que obtienen de las orientaciones mercantiles de sus explotaciones, el programa agroambiental les resulta poco atractivo a los grandes agricultores como complemento de renta. Si se adhieren al mismo, lo hacen más por razones ecológicas inspiradas en una especie de capitalismo verde, ya que su reproducción social como agricultores no se ve amenazada en el actual contexto de cambios que experimenta la agricultura.

En los pequeños agricultores, por el contrario, se manifiesta con mayor nitidez el predominio de la dimensión económica, al ser conscientes de las dificultades de mantener su actual nivel de renta con las orientaciones productivas tradicionales. Por eso, el escaso atractivo de los programas agroambientales para los pequeños agricultores radica en la reducida cuantía de las ayudas en comparación con otras procedentes de la PAC, como las de superficie. Los casos de optar por el programa de agricultura ecológica confirman esta tesis del predominio de la dimensión económica, si bien con la particularidad de que aquí prima una componente de esta dimensión que no es sólo la de complementar rentas, sino también la de esperar obtener mayor rentabilidad que con las orientaciones tradicionales. Sólo en grupos minoritarios, que pueden ser catalogados como una élite más o menos ilustrada formada 
por agricultores que no tienen una dedicación principal a la agricultura, puede observarse la presencia de una dimensión ambiental en su decisión de optar por este programa agroambiental.

En tercer lugar, que la presencia de la dimensión ambiental se hace dominante allí donde los efectos negativos de la agricultura sobre el medio ambiente se muestran de modo tangible. Por ejemplo, en zonas donde se manifiestan de modo palpable tales efectos y sobre los que existe un consenso generalizado de que es necesario reorientar la actividad agraria, la dimensión ambiental se hace presente en las decisiones de los agricultores.

En cuarto lugar, la investigación realizada nos lleva a afirmar que es más fácil modificar los comportamientos de los agricultores, que sus actitudes respecto a la política agroambiental. Hemos encontrado agricultores que se han acogido a algún programa agroambiental sólo por razones instrumentales de carácter económico, sin haber mostrado actitudes favorables, y que, sin embargo, una vez desarrolladas sus nuevas prácticas, les han hecho modificar su actitud respecto a la utilidad e interés de la política agroambiental.

En quinto lugar, y como corolario de lo anterior, el análisis efectuado pone de manifiesto la influencia - mayor o menor, según los casos- de los elementos que componen la estructura de oportunidades sobre las decisiones de los agricultores, influencia que se convierte en decisiva al incidir sobre el comportamiento de los agricultores. En efecto, la cuantía de la ayuda es un elemento decisivo para hacer que los agricultores se acojan al programa, independientemente de que su actitud sea o no favorable a la existencia de una política agroambiental. Asimismo, la presencia de una estructura administrativa de servicios cercana a los agricultores para prestarles el asesoramiento que necesiten al optar por programas que implican cambios en la gestión de sus explotaciones, es otro elemento decisivo. Igualmente, las actividades de formación y divulgación de los programas agroambientales, ya sea realizadas por las organizaciones profesionales o por la propia Administración pública, constituyen otro elemento clave por sus efectos sobre el comportamiento de los agricultores.

\section{Bibliografía}

ALLEN, P. y otros (1991). «Integrating social, environmental, and economic issues in sustainable agriculture». American Journal of Alternative Agriculture, vol. 6, n. ${ }^{\circ}$ 1, p. 34-39.

ARÁmbarRi, P. (1994). «Los nuevos ecologistas». Arbor CXLIX, 585, p. 103-123. BAGER, T.; PROOST, J. (1997). «Voluntary regulation and farmers' environmental behaviour in Denmark and The Netherlands". Sociologia Ruralis, 37 (1). p. 77-96. Bager, T.; SøgaARD, V. (1994). The farmer and the environment. Attitudes and behaviour investigated through a questionnaire. Esbjerg: SUC.

BALDOCK, D.; LOWE, P. (1996). «The development of European Agri-environment Policy». En: Whitby, M. The European Environment and CAP Reform: Policies and Prospects for Conservation. Wallingford, UK: CAB International, p. 8-26.

BeOpOUlos, N.; SKURAS, D. (1997). «Agriculture and the Greek Rural Environment». Sociologia Ruralis, vol. 37, n. ${ }^{\circ}$ 2, p. 255-269. 
Boudon, R. (1981). La lógica de lo social. Madrid: Rialp.

BROTHERTON, I. (1989). «Farmer participation in voluntary land diversion scheme: Some observations from theory». Journal of Rural Studies, n. ${ }^{\circ}$ 5, p. 299-304.

- (1991). "What Limits participation in ESA?». Journal of Environmental Management, 32, p. 241-249.

BROWN, S. (1990). "Humans and their environment: changing attitudes». En Silvertown, J.; SARRE, P. H. (eds.): Environment and society. Londres: The Open University, p. 238-269.

Buller, H.; HOGgarT, K. (1998). «Pluralidad e implementación de políticas de medio ambiente en la Unión Europea». Revista Internacional de Sociología, n. ${ }^{\circ} 19$ y 20. CSIC, p. 153-179.

Buller, H.; Wilson, G.A.; Höll, A. (ed.) (2000). Agri-environmental Policy in the European Union. Ashgate, p. 291.

BuTTEL, F.H. (1996). «Instituciones sociales y cambio ambiental: cuestiones teóricas recientes». Información Comercial Española, n. ${ }^{\circ}$ 751, Madrid, p. 105-118

Canuto, J.C. (1998). Agricultura ecológica en Brasil. Perspectivas socioecológicas. Tesis doctoral. Universidad de Córdoba.

COMISIÓN DE LAS COMUNIDADES EUROPEAS (1997). Informe de la Comisión al Consejo y al Parlamento Europeo sobre la aplicación del Reglamento 2078/92. COM (97) 620 final, Bruselas.

Costabeber, J. A. (1998). Acción colectiva y proceso de transición agroecológica en Río Grande do Sul (Brasil). Tesis doctoral. Universidad de Córdoba.

DALY, H.E.; GAYO, D. (1995). «Significado, conceptualización y procedimientos operativos del desarrollo sostenible: posibilidades de aplicación a la agricultura». En CAdenas, A. (ed.). Agricultura y desarrollo sostenible. Madrid: Serie Estudios del MAPA, p. 19-38.

Daugbjerg, C. (1997). «Reforming the CAP: The roles of Policy Networks and Broader Institutional Structures». ALF Working Paper. Esbjerg (Dinamarca): South Jutland University Centre.

DOERING, O. (1992). «Federal policies as incentives or disincentives to ecologically sustainable agricultural systems». En Olson, R.K. (ed.). Integrating sustainable agriculture, ecology, and environmental policy. Nueva York: The Haworth Press, p. 21-36.

Euopean Commission, DG VI (1997). Agriculture and Environment. BruselasLuxemburgo: CAP Working Notes.

FrancisCO, A. de (1997). Sociología y cambio social. Barcelona: Ariel Sociología.

Frouws, J. (1998). "The contested redefinition of the countryside. An analysis of rural discourses in the Netherland». Sociologia Ruralis, vol. 38, n. ${ }^{\circ}$, p. 54-68.

GARRIDO, F. (2001). La cuestión ambiental en la agricultura: actores sociales y política agroambiental en España. Málaga: Fundación UNICAJA, 323 p.

GARRIDO, F.; MOYANO, E. (1994). EC and National Regulations on Environment and Agriculture in Denmark, the Netherlands and Spain. Report 1, n. ${ }^{\circ}$. EV5V-CT920162. Esbjerg (Dinamarca).

GLIGO, N. (1990). «Los factores críticos de la sustentabilidad ambiental del desarrollo agrícola». Comercio Exterior, vol. 40, n. ${ }^{\circ}$ 12. Méjico, p. 1135-1142.

Gómez Benito, C.; Paniagua Mazorra, A. (1996): «Caracterización sociodemográfica de la sensibilidad ambiental de España». Información Comercial Española, n. ${ }^{\circ}$ 751, marzo. Ministerio de Comercio y Turismo. 
Guía Metodológica del ObServatorio EuRopeo LEADER (2001). La Declaración de Cork: "Por un mundo rural vivo». Conferencia Europea de Desarrollo Rural, Cork (Irlanda). 7 a 9 de noviembre de 1996.

Just, F. (1997). Does «soft» regulations matter? Working Paper. Esbjerg: Department of Cooperative and Agricultural Research, SUC.

- (1998). Voluntary regulations and agri-environment. Working Paper. Esbjerg: Department of Cooperative and Agricultural Research, SUC.

LOWE, P. (1997). «European perceptions of the CAP and the agrienvironmental policy». Ponencia presentada en el I Encuentro Internacional de Primavera sobre Desarrollo Rural. Universidad de Córdoba, 21 al 25 de abril, Córdoba.

McHenRY, H. (1996). «Farming and environmental discourses: A study of the depiction of environmental issues in a german farming newspaper». Journal of Rural Studies, vol. 12, n. ${ }^{\circ}$, p. 375-386.

MiCHELSEN, J. (1994). Introducing EC Regulations on agriculture and environment in Denmark. A change of agricultural discourse?, n. ${ }^{\circ}$ 32/94. Esbjerg: Department of Cooperative and Agricultural Research. South Jutland University Center.

Ministerio de Agricultura, Pesca y Alimentación (1994). Programas de Ayudas para Fomentar Métodos de Producción Agraria Compatibles con las Exigencias de la Protección y la Conservación del Espacio Natural, 3 volúmenes, Madrid.

MORRIS, C.; POTTER, C. (1995). «Recruiting the new conservationists: Farmers' adoption of agri-environmental schemes in the UK». Journal of Rural Studies, n. ${ }^{\circ} 11$, p. 51-63.

Moyano, E.; Paniagua. A. (1998). «Agricultura, espacios rurales y medio ambiente». Revista Internacional de Sociología (RIS), n. ${ }^{\circ} 19$ y 20, CSIC, p. 127-152.

OÑATE, J. J.; ÁlvareZ, P. (1997). «El programa de Estepas Cerealistas en Castilla y León». Revista Española de Economía Agraria, n. ${ }^{\circ} 179$.

Patricio, T.; Valadas, A.; SAMPaio, E. (1995). The attitudes of Portuguese farmers towards agri-environmental measures: some results of an empirical study. Ponencia presentada al XVI European Congress of Rural Sociology. Agosto, Praga.

PRIMDAHL, J.; HANSEN, B. (1993). «Agriculture in Environmentally Sensitive Areas: Implementing the ESA Measure in Denmark». Journal of Environmental Planning and Management, vol. 36, n. ${ }^{\circ}$ 2, p. 231-238.

REDCLIFT, M. (1991). "The multiple dimensions of sustainable development». Geography, n. ${ }^{\circ}$ 76, 330, p. 36-42.

VILADOMIU, L.; Rosell, J. (1996). Informe preliminar sobre el Programa de Compensación de Rentas en los regadios de la Mancha Occidental y Campo de Montiel. Universidad Autónoma de Barcelona.

WILSON, G.A. (1992). «A survey on attitudes of landholders to native forest on farmland». Journal of Environmetal Management, n. ${ }^{\circ} 34$, p. 117-136.

- (1996). «Farmer Environmental Attitudes and ESA Participation». Geoforum, vol. 27, n. ${ }^{\circ}$ 2, p. 115-131.

- (1997). «Factors influencing farmers participation in the Environmentally Sensitive Areas Scheme». Journal of Environmental Management, n. ${ }^{\circ}$ 50, p. 67-93. 